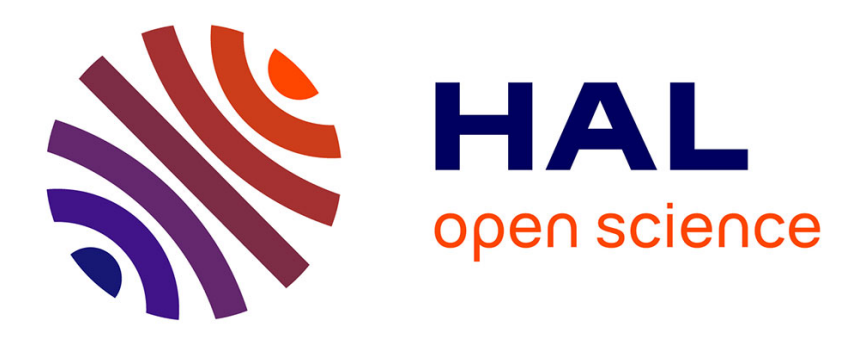

\title{
A state variable description of mechanical properties
}

D. Stone, Che-Yu Li

\section{To cite this version:}

D. Stone, Che-Yu Li. A state variable description of mechanical properties. Revue de Physique Appliquée, 1988, 23 (4), pp.639-647. 10.1051/rphysap:01988002304063900 . jpa-00245812

\section{HAL Id: jpa-00245812 https://hal.science/jpa-00245812}

Submitted on 1 Jan 1988

HAL is a multi-disciplinary open access archive for the deposit and dissemination of scientific research documents, whether they are published or not. The documents may come from teaching and research institutions in France or abroad, or from public or private research centers.
L'archive ouverte pluridisciplinaire HAL, est destinée au dépôt et à la diffusion de documents scientifiques de niveau recherche, publiés ou non, émanant des établissements d'enseignement et de recherche français ou étrangers, des laboratoires publics ou privés. 


\title{
A state variable description of mechanical properties
}

\author{
D. Stone and Che-Yu Li
}

Department of Materials Science and Engineering, Cornell University, Bard Hall, Ithaca, NY 14853-1501, U.S.A.

(Reçu le 15 juin 198\%, accepté le 9 novembre 198ý)

\begin{abstract}
Résumé.-Une approche utilisant des variables d'état pour décrire la déformation inélastique d'un solide cristallin est traftée. Cette approche est basée sur la formulation developpée par Hart [1]. Nous démontrons qu'elle décrit des processus de déformation communément observés tels que le fluage. Par contre, la description d'autres processus tels que: déformation inhomogène ou influence de l'interaction entre dislocations et atomes en solution sur le processus de déformation va au-delà. du but recherché par cette théorle.
\end{abstract}

Abstract.--A state variable approach based on Hart's [1] formulation for describing nonelastic deformation of crystalline solids is reviewed. It is shown to describe commonly observed deformation phenomena, such as creep. Presently, deformation phenomena such as inhomogeneous flow and those involving strong dislocation-solute interactions are not within the scope of this approach.

The nonelastic properties of a crystalline solid depend upon prior deformation and thermal history. To fully account for the history effects can be a cumbersome task in stress analysis and mechanical testing. If the deformation properties can be demonstrated to be uniquely characterized by state variables, and if the manner in which the properties evolve along an arbitrary thermal or mechanical path can be shown to depend upon the state variables as well, time dependent stress analysis and materials testing can be greatly simplified.

The appropriate state variables are not easily identifled based solely on theoretical arguments. The experimental effort required to establish them can also be difficult. In part this is because a varlety of mechanisms contribute to nonelastic deformation, and the delineation of these mechanisms requires extensive experimental effort. For example, the separation of the grain boundary sliding contribution from grain matrix deformation during creep at elevated temperatures is an important but difficult task.

This paper reviews a phenomenological approach, originally proposed by E.W. Hart designed to establish state variable flow relationships based on direct experimental measurements without first resorting to a microscopic theory of deformation [1]. Many of the state variables involved in this approach have been given physical significance, but no general theory based on microscopic processes has been developed.
The existence of state variables characterizing the structural state of a material is a fundamental basis of Hart's formulation. In this approach the load relaxation test has been used extensively to determine flow relations at a constant (or nearly constant) structure. It has been found that the logarithmic plots of stress versus strain rate; obtained from load relaxation tests of specimens with different degrees of workhardening, can be translated along a linear path to overlap each other. This is the so-called scaling relationship, not hitherto predicted by dislocation based theories [1]. Mathematically it means that the plots belong to a one-parameter family of curves, a concept first proposed by E.W. Hart. This parameter is called the hardness, $\sigma *$, and is a state variable. It is uniquely defined by the current value of stress, strain rate, and temperature, which are other state variables. In practice, it is not only important to know the existence of $\sigma^{*}$, but also to be able to experimentally measure this parameter.

In contrast, often-used flow laws relating minImum creep rate to applied stress are obtained experimentally without the assurance that the stress dependence of the creep rate corresponds to that of a constant structural state. In fact, such a correspondence is highly unlikely. To interpret the minimum creep rate data additional information on the structural state of the material and its variation during creep are required. 
Flow stress-strain rate data obtained in load relaxation tests covering a wide range of strain rates and temperatures are 111 ustrated in $10 \mathrm{~g}-10 \mathrm{~g}$ form in Figures 1 and $2[2,3]$. One striking feature of these data is that the strain rate sensitivity, as defined by $d \log \sigma / d \log \dot{\varepsilon}$, varies with temperature and stress. Superficially, the load relaxation data differ significantly from creep data presented in familiar power law form. A purpose of this paper is to demonstrate that the constant structure load relaxation data in Figures 1 and 2 are consistent with deformation data obtained in other experiments. A good example is the creep data reported in the form of creep maps, as shown in Figure 3 [4].

It is important to note that, in addition to constant structure load relaxation data that specifies $\sigma *$ and the flow relations, one also requires a workhardening law to establish the evolution of $\sigma *$ along an arbitrary mechanical path [5]. A workhardening law does not a priori involve the state variables $\sigma^{*}$ and $\sigma$. If the workhardening relationship is not a sole function of state variables, it will not be easy to integrate the deformation equations along an arbitrary path in a stress analysis. In this case, experiments would be required to determine the workhardening behavior for each deformation path.

\section{A State Variable Approach}

The most basic phenomenon to have been characterized by state variables is time-dependent,

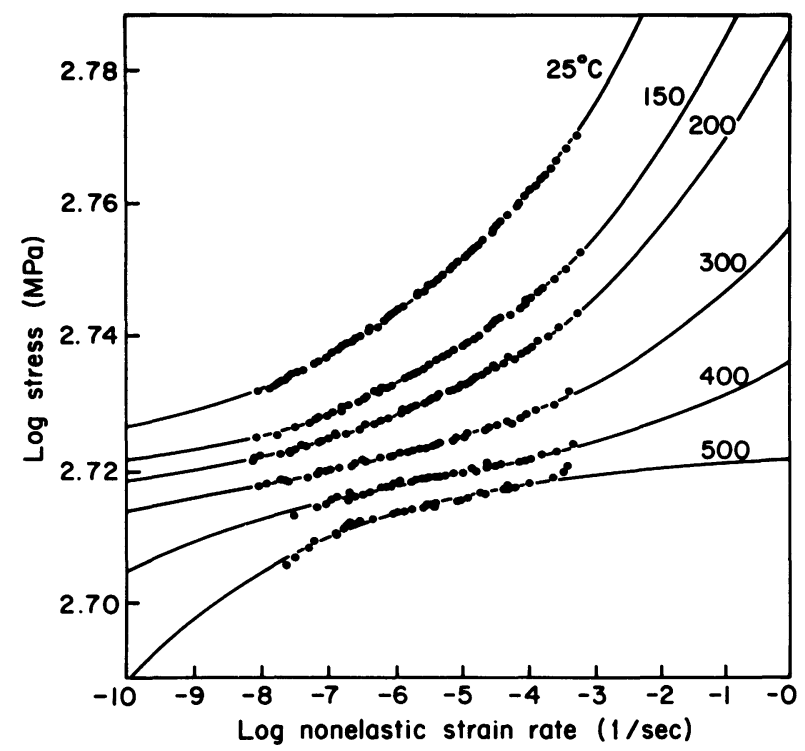

Figure 1. Constant structure load relaxation data of 316 Stainless Steel at several temperatures. The strain rate sensitivity is seen to be a function of strain rate and temperature. After ref. [2].

plastic deformation in polycrystalline metals involving grain matrix processes only. The experimental basis for establishing the state variables pertaining to plastic deformation will be reviewed initially.

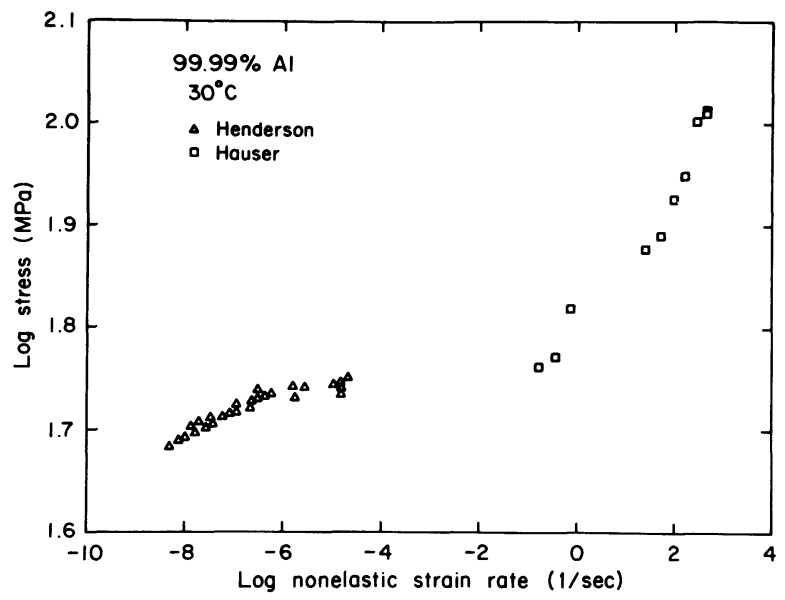

Figure 2. Load relaxation data of $\mathrm{Al}$ at constant structure, after ref. [3].

Hart [6] first realized that the load relaxation experiment can be used to obtain flow stressstrain rate data at a constant structure. Such data usually cover a five decade strain rate window with minimal scatter, revealing the characteristic shapes of flow curves [7]. To begin a load relaxation run, the specimen is loaded at a constant displacement rate, preferably in a screw driven machine, to a predetermined level of load. The crosshead is then fixed and the load allowed to relax as the specimen continues to deform. As the load relaxes the strain rate decreases. The rate of load relaxation is related to the plastic strain rate of the specimen through the machine constant, or stiffness. For a machine of high stiffness, the total elongation required to sig-

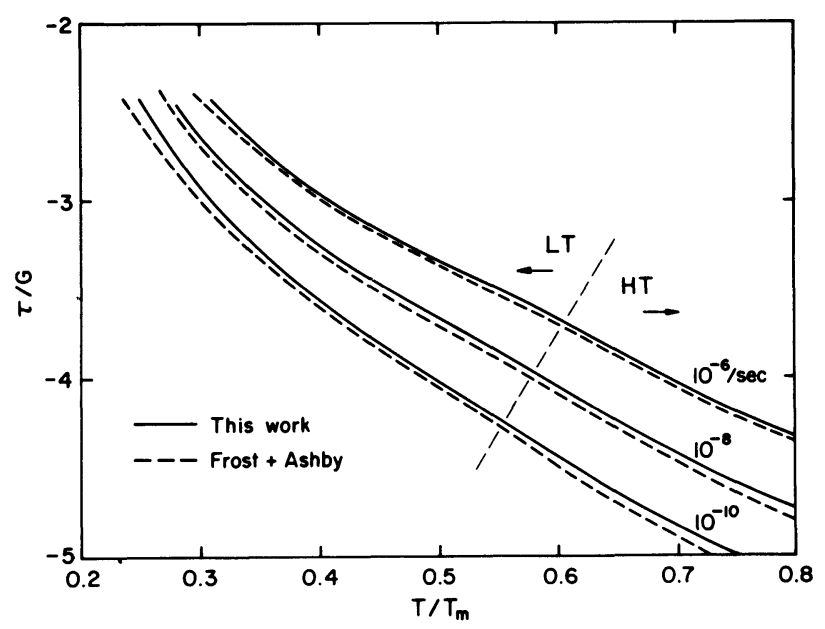

Figure 3. Constant shear strain rate contours of Ni for steady state-1ike (SSL) power law creep. See ref. [4].

nificantly relax the load is sma11, on the order of $0.1 \%$ to $0.3 \%$, such that little workhardening occurs. Thus, the specimen is able to maintain a nearly constant structural state provided timedependent processes, such as thermal recovery and strain aging, are not important. 
In the case that structural changes or changes in the rate parameters are suspected to play a role, one can perform a reloading experiment to detect any time-dependent change in the structural state [6]. The reloading is made to a level of stress below the initial stress of the previous run. If, after a transient period, the reloading data are found to converge onto the previous load relaxation curve, one may conclude that the structural state or the rate parameters have remained unchanged during the initial relaxation run. A1though the load relaxation test does not guarantee constant structure flow data, the reloading relaxation run can be used to determine whether time dependent effects are present. Flgures 4 and 5 show inftial and repeated load relaxation data of Ferrovac-E Iron and an $\mathrm{Fe}-0.10 \% \mathrm{~T} 1$ alloy, respectively, at $\mathrm{T}=30^{\circ} \mathrm{C}[8]$. In the former case the reloading data do not approach the initial load relaxation data at low strain rates. This is believed to be caused by the effects of strain aging

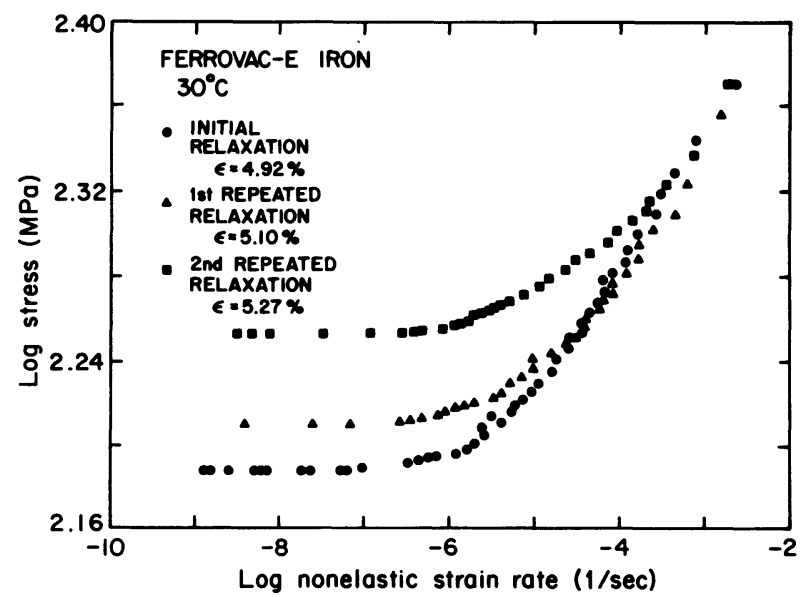

Figure 4. Loading and reloading relaxation data of Ferrovac-E Iron demonstrating the case where reloading data do not overlap initial relaxation data due to strain aging. After ref. [8].

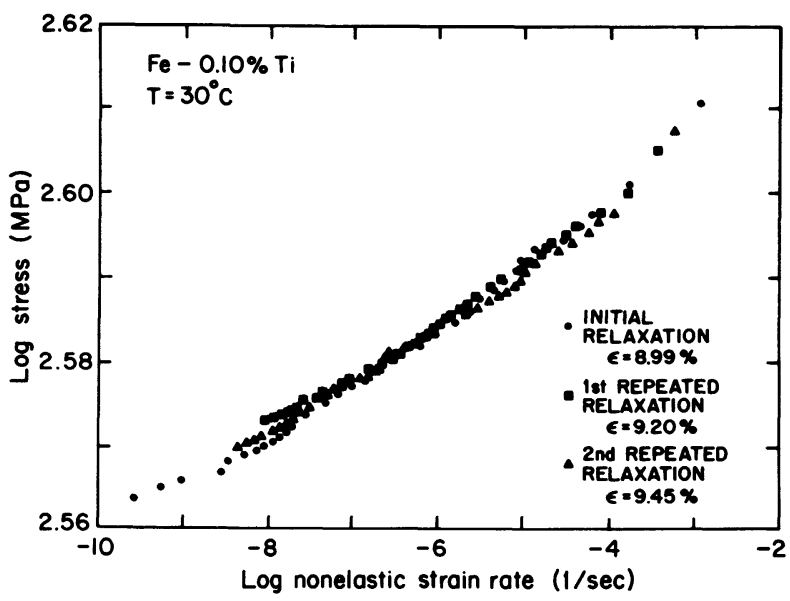

Figure 5. Loading and reloading relaxation data of $\mathrm{Fe}-0.10 \% \mathrm{Ti}$ alloy. These data demonstrate the case where the structure of the material remain unchanged throughout relaxation. After ref. [8]. during load relaxation. In the case of the Fe$0.10 \% \mathrm{TI}$ alloy, the reloading data fall we11 on top of the inftial relaxation data suggesting the absence of time-dependent changes.

The availability of constant structure load relaxation curves with their clearly defined shapes have allowed the discovery of the scaling relationship. Figures 6 and 7 are typical load relaxation data obtained at a high and low homologous temperature, respectively $[9,10]$, exhibiting scaling in that the individual curves can be rigidly translated along a straight line to overlap one another.

The high homologous temperature data, Figure 6 , show a characteristic concave downward shape whereas the low temperature data are concave upward in shape. Composite curves resulting from translations according to the scaling relationships are depicted in Figures 8 and 9 . Because of scaling, the curves in Figures 8 and 9 can be considered to belong one parameter families of curves. This parameter is the hardness parameter, $\sigma *$, which is uniquely specified by a constant structure load relaxation curve. Mathematically, each relaxation curve is represented by a state variable relationship $\sigma *=f(\sigma, \dot{\varepsilon})$ such that $\dot{\varepsilon}$, the plastic strain rate and $\sigma$, the applied stress, are established to be state variables. For convenlence, $\sigma^{*}$ is measured as the high strain rate limiting stress of the high homologous temperature curves or the low strain rate limiting stress of the low homologous temperature curves [2]. For a material at a constant structure, $\sigma^{*}$ has the same value for both the high homologous temperature data and the low homologous temperature data. It is a scalar and depends weakly on temperature such that $\sigma * / G$ is independent of temperature, where $G$ is the shear modulus. Physically, $\sigma *$ reflects the dislocation cell structure, and has been linked to the dislocation cell size through a Hall-Petch type relationship [9].

At intermediate temperatures, such as for the $300^{\circ} \mathrm{C}$ curve in Figure 1 , simple scaling is not possible because both high and low temperature modes of deformation contribute and the scaling direction is different for each mode [2].

For a wide range of crystalline solids, including ceramics, constant structure load relaxation data are generally found to display two basic deformation characteristics, depending, as 11lustrated in Figures 8 and 9, on the homologous tem-

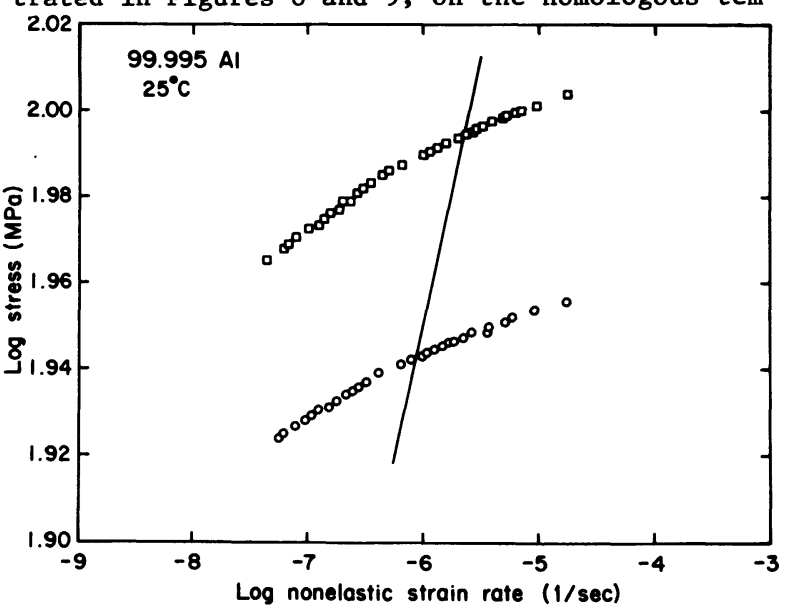

Figure 6 . Load relaxation data of $A 1$ at two levels of $\sigma^{*}$, demonstrating the scaling direction at high homologous temperatures. The load relaxation data exhibit a concave downward shape. After ref. [9]. 


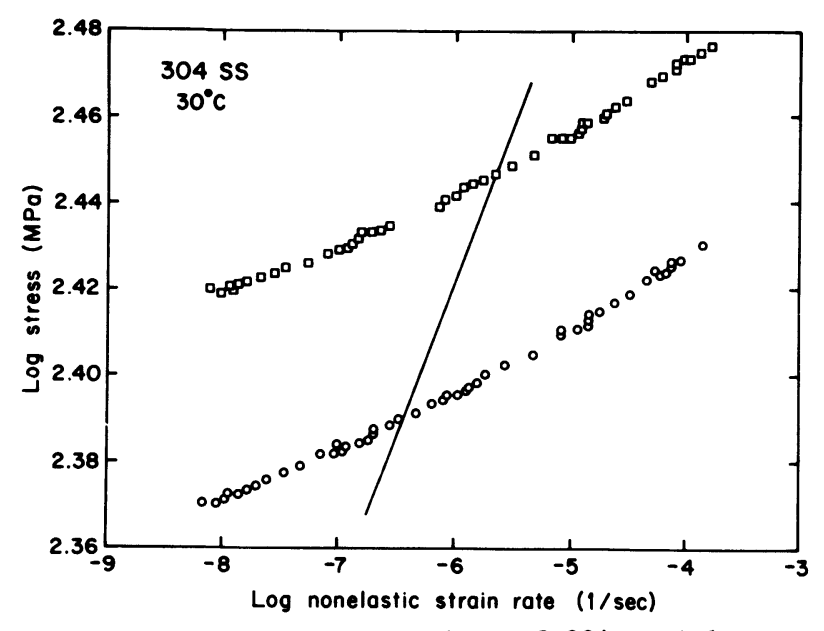

Figure 7. Load relaxation data of 304 Stainless steel at two levels of $\sigma *$, demonstrating the scaling behavior for low homologous temperatures. The curves are concave upward. After ref. [10].

perature. Hart has associated the h1gh homologous temperature behavior with dislocation climb and the low homologous temperature behavior with dislocation glide and has proposed a deformation mode1 $[1,11]$. In this model the driving force for dislocation climb is $\sigma_{a}$, which is a state variable and is close to the applied stress at high homologous temperatures. For dislocation glide, $\sigma_{f}$ is

the driving force, which is the difference between the applied stress and $\sigma_{a}$. The flow equations have the forms:

$$
\begin{aligned}
& \ln \left(\frac{\sigma^{*}}{\sigma_{a}}\right)=\left(\frac{\dot{\varepsilon} *}{\dot{\varepsilon}}\right)^{\lambda} \\
& \dot{\varepsilon}=\dot{a} *\left(\frac{\sigma_{f}}{G}\right)^{M}
\end{aligned}
$$

for high and low homologous temperatures, respectively, where $\varepsilon^{*}$ and $a^{*}$ are rate parameters, and $\lambda$ and $M$ are pure numbers. At low homologous temperatures or high strain rates, $\sigma_{a} \cong \sigma^{*}$. The scaling

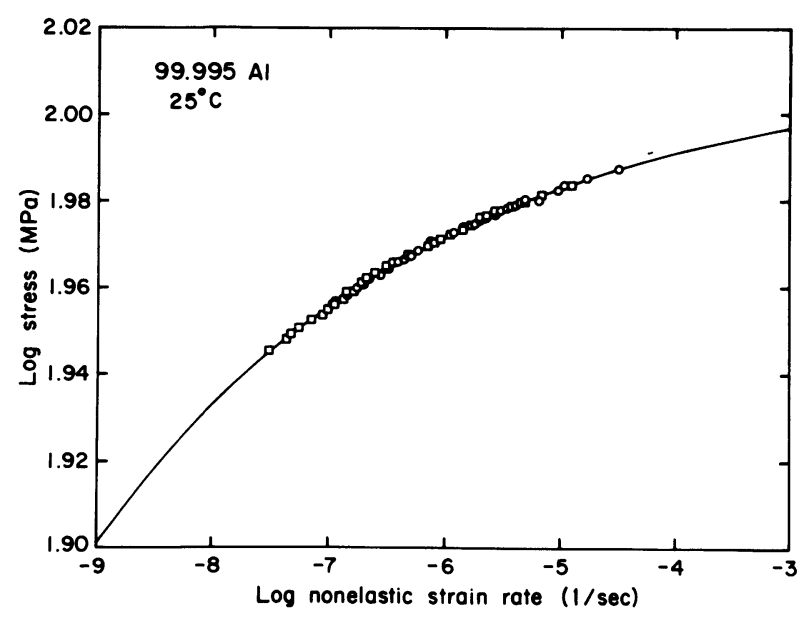

Figure 8. Composite curve of Aluminum constructed based on the translation of the load relaxation data in Figure 6 along the scaling direction. The solid curve has the form of Equation 2, with $\sigma *=$ $103 \mathrm{MPa}, \dot{\varepsilon}^{*}=1.1 \times 10^{-13} / \mathrm{sec}$, and $\lambda=0.15$.

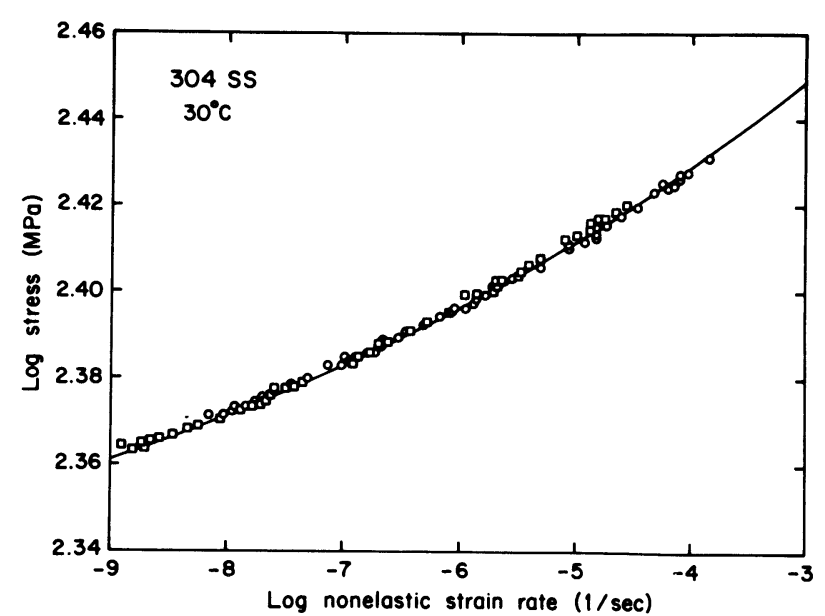

Figure 9. Composite curve of 304 SS constructed based on the translation of the load relaxation data in Figure 7 along the scaling direction. The solid curve has the form of Equation 1, with $\sigma^{*}=$ $199 \mathrm{MPa}, \dot{a} *=9 \times 10^{38} / \mathrm{sec}$, and $M=14$.

relationship for high homologous temperatures is found experimentally to be of the form:

$$
\dot{\varepsilon}^{*}=\dot{\varepsilon}_{0}^{*}\left(\frac{\sigma^{*}}{\mathrm{G}}\right)^{\mathrm{m}} \exp \left(\frac{-\mathrm{Q}}{\mathrm{RT}}\right)
$$

Here, $\dot{\varepsilon}^{*}$ is a frequency factor, $m$ is a constant, which is found experimentally to be about 5 for several metals $[1,12]$. $Q$ is experimentally observed to correspond to the activation energy for diffusion. Depending on temperature, $Q$ may vary reflecting elther volume diffusion or pipe diffusion as the dominant mechanism. The temperature dependence of $\dot{a}^{*}$ at low homologous temperatures is found to be weak compared to that of $\dot{\varepsilon}^{*}$ [2].

The slope of the scaling path, the line along which the load relaxation curves can be translated to overlap one another, is $1 / \mathrm{m}$ for Equation 1 and $1 / \mathrm{M}$ for Equation 2 . The high homologous temperature scaling can be verified by noting that $\mathrm{d} \log \dot{\varepsilon} * / \mathrm{d} \log \sigma *=\mathrm{m}$. A similar consideration shows that $1 / M$ is the slope of the scaling path at low homologous temperatures. The shape factor $\lambda$, which, mathematically, determines how sharply concave downward the high homologous temperature curve turns at low strain rates, appears to be a constant with a value of approximately 0.15. The physical significance of this number remains to be examined. $M$, which defines the shape of the low homologous temperature data, is generally found to vary between 7 and 20 [12].

At high homologous temperatures, the strain rate becomes singular as $\sigma_{a}$ approaches $\sigma^{*}$. This singularity can pose difficulties during numerical integration. The functional form of Equation 1 appears, however, to have fundamental significance, so that caution should be exercised when attempting to modify the form of Equation 1 for numerical convenience.

The form of Equation 1 is not accountable based on current single dislocation based deformation theories. Although the detailed mechanisms remain to be developed, it is apparent that this form is insensitive to detailed atomic and dislocation structures. This points to deformation processes involving collective motion of dislocations with well defined statistics [13]. 
The hardness parameter, $\sigma *$ will increase as a result of deformation. To describe this evolution along an arbitrary mechanical path requires a workhardening relationship that can not be assumed, without experimental verification, to be a function solely of state variables $\sigma *$ and $\sigma_{a}$. Independent experiments have been performed, however, to demonstrate the adequacy of these state variables to describe workhardening $[5,14]$. The form of the workhardening law is established based on data obtained from a combination of load relaxation and tensile tests.

The absolute workhardening parameter, defined as :

$$
\left.\Gamma \equiv \frac{\mathrm{d} \ln \sigma *}{\mathrm{~d} \varepsilon}\right|_{\dot{\varepsilon}}
$$

has been demonstrated to be of the form $[5,11]$ :

$$
\Gamma=\left(\frac{\mathrm{A}}{\sigma^{*}-\sigma_{0}}\right)^{\delta}\left(\frac{\sigma \mathrm{a}}{\sigma^{*}}\right)\left(\mathrm{A} /\left(\sigma^{*}-\sigma_{0}\right)\right)
$$

where $A$ and $\sigma_{0}$ are constants and $\delta$ is a pure number. At low homologous temperatures, when deformation is controlled by glide-friction, $\sigma_{a} / \sigma^{*} \cong 1$. It can be shown that, for $\sigma_{0} \cong 0$, at these temperatures $1 / \delta$ is the conventional strain hardening exponent. Based on the Considère condition, the value of $\sigma^{*}$ is $A$ when a specimen begins to neck in a tensile test.

At high homologous temperatures $\Gamma$ becomes sensitive to strain rate, which is viewed as a manifestation of dynamic recovery. An explicit rate dependence can be obtained by substituting the expression for $\sigma_{a} / \sigma *$ in terms of $\dot{\varepsilon} * / \dot{\varepsilon}$ based on Equation 1. If we define $\Gamma *=\left(A /\left(\sigma *-\sigma_{0}\right)\right)^{\delta}$ and $\dot{\alpha} *$ $=\dot{\varepsilon} *(\Gamma *)^{1 / \delta \lambda}$, Equation 5 becomes [5]:

$$
\ln \frac{\Gamma *}{\Gamma}=\left(\frac{\dot{\alpha}}{\dot{\varepsilon}}\right)^{\lambda}
$$

Like the hardness parameter, $\sigma *, \Gamma *$ is an athermal term and $\dot{\alpha} * / \dot{\varepsilon}$ gives the rate dependence of $\Gamma$. At high strain rates, $\Gamma \cong \Gamma^{*}$, which governs workhardening under conditions where glide friction controls. The form of the workhardening function (Equation 6) is consistent with current concepts on workhardening that the rate independent part reflects the mechanical effects alone, whereas the rate dependent part results from the effects of dynamic recovery [5].

\section{Applicabllity of the State Variable Approach}

The yield stress of a polycrystalline solid is often reported as a function of temperature. These types of data correspond to those represented by equations 1 and 2 with the appropriate activation energies. This is a straightforward comparison [12]. Sometimes the ultimate tensile stress is reported. In this case, at least for low homologous temperatures, the Considère criterion can be used based on the workhardening correlation. For creep data, a somewhat more involved discussion will have to be made.
In principle, the flow relationships may be integrated to describe plastic flow along an arbitrary deformation path. In fact this has been done for a variety of situations $[12,15]$. These detailed analyses are outside the scope of the present paper. Instead, a part of a deformation map created by Frost and Ashby [4] will be examined without resorting to a detailed analysis to illustrate the applicability of the present state variable approach. In particular, we are interested in the power law creep regime of the deformation map for nickel, Figure 3. Fach curve of the map in this regime is the locus of temperature and flow stress required to produce a particular steady state-like (SSL) creep rate. Above about $\mathrm{T} / \mathrm{T}_{\mathrm{m}}=0.6$, where $\mathrm{T}_{\mathrm{m}}$ is the absolute melting temperature, the data shown correspond to a SSL creep rate having a fifth power dependence on stress and an activation energy of volume self-diffusion. Below about $0.5 \mathrm{~T} / \mathrm{T}_{\mathrm{m}}$ a higher stress exponent is observed along with an activation energy corresponding to pipe diffusion. The stress dependence at low temperatures reflects, in part, that of pipe diffusion (a $\sigma^{2}$ dependence [4]). By excluding this effect, therefore, in both temperature ranges the SSL creep data can be considered to have the same stress dependence.

The fifth power stress dependence of the SSL creep rate coincides with the stress exponent of the scaling relationship, Equation 3 at high homologous temperatures. This correspondence can be taken as a convenient starting point for the discussion.

We note that along the scaling direction, $\sigma_{a} / \sigma *$ and $\dot{\varepsilon} / \dot{\varepsilon} *$ are constants. The form of the scaling law is the same as that for power law creep, suggesting that SSL creep occurs for a value of $\sigma / \sigma *$, that is (at least to a close approximation) insensitive to temperature and strain rate. Thus, at higher applied stresses, higher values of $\sigma^{*}$. operate during SSL creep. If we substitute $\dot{\varepsilon} * / \dot{\varepsilon}=$ constant into the workhardening relationship, Equation 6 , we obtain an expression relating $\Gamma$ to $\Gamma *$ at SSL creep

$$
\ln \left(\frac{\Gamma *}{\Gamma}\right)=\text { const. } \Gamma^{*} 1 / \delta
$$

The above equation, along with the workhardening relationships, Equations [5] and [6], can be used to examine how SSL creep is established. Figure 10 shows typical creep curves for an annealed material at high $\left(\sigma>>\sigma_{1}^{*}\right)$, intermediate $\left(\sigma \cong \sigma_{1}^{*}\right)$, and low $(\sigma<<\sigma *)$ initial stresses respectively $\left(\sigma \sigma_{1}^{*}\right.$ is the initial hardness). According to the present view, the shapes of these curves--that is, the extent of primary creep--is determined mainly by the workhardening relationship and does not require the presence of thermally induced, static recovery. During the primary creep regime, the specimen workhardens so that the value of $\sigma^{*}$ increases. According to Equations (5) and (6), the rate of work hardening decreases at high $\sigma^{*}$ leading to a SSL behavior. This process can be verified by numerical integration of Equations 1, 2, and 5 to simulate experimental data $[12,15,16]$.

Consider that, in Figure 10, the initial hardnesses, $\sigma_{1}^{*}$, of the three specimens are the same, near the level of the intermediate stress. The 
high stress is of the order of A in Equation 5 . For a high applied stress, the initial $\Gamma^{*}$ is large because of the low initial $\sigma^{*}$. According to Equation 6 , the initial value of $\Gamma$ is large also because of the high initlal strain rate. Thus, initially $\sigma *$ increases rapldly, which reduces $\dot{\varepsilon}$. As the rate of work hardening decreases, a SSL creep behavior results. At SSL creep, $\Gamma / \Gamma^{*}$ is large, but $\Gamma *$ is significantly lower than its initial value. A large transient stage, or primary creep, 1s expected before the occurrence of SSL creep because of the large increase in $\sigma *$.

At an intermediate applied stress, the inftial value of $\Gamma$ is smaller because of a lower initial stress or strain rate. Thus the value of $\sigma *$ never becomes very large. The value of $\Gamma$ will, however, become sufficiently low to cause SSL behavior, but here the final value of $\Gamma / \Gamma *$ (according to Equation 7) is small as $\Gamma *$ remains high due to low $\sigma *$ The small change in $\sigma^{*}$ leads to a shorter transient stage as experimental data demonstrate [17].

At the lowest stress (assuming this stress is sufficiently low) $\Gamma$ is not large enough to produce appreciable workhardening. Because the material can not workharden, this behavior falls outside the power law creep regime with an exponent of 5 .

Mathematically, the concept of steady state creep requires that the rate of change in the hardness parameter becomes zero. Indeed, SSL creep behavior may be consistent with the rate of workhardening, $\Gamma$, being low. During SSL creep at

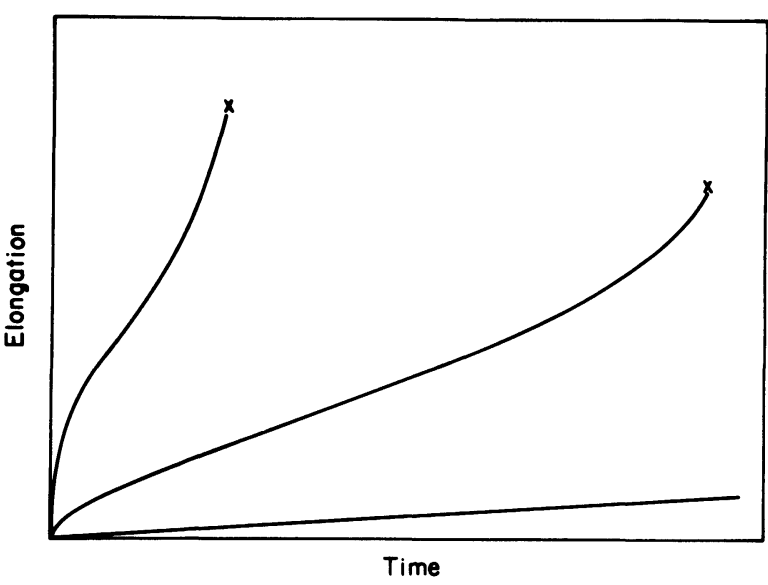

F1gure 10. Typical high homologous temperature creep curves at high, Intermediate, and low levels of stress.

high stresses the workhardening rate is near $\Gamma *$, but $\Gamma^{*}$ is low because $\sigma^{*}$ is high. At intermediate stresses $\Gamma^{*}$ is high because $\sigma^{*}$ is low, but the rate of workhardening is low because $\Gamma / \Gamma^{*}$ is low. This type of compensating effect, which does not require the ald of static recovery, is apparently consistent with the power law creep behavior exhibited by the nickel data.

In Figure 3, the dashed curves represent the flow stress as a function of temperature, at constant strain rate, with $\sigma / \sigma *=0.5$ substituted Into Equation 3 . This value of $\sigma$ corresponds to $\dot{\varepsilon} / \dot{\varepsilon} *=115$. Actual values of $\sigma \%$ and $\dot{\varepsilon} *$ were established at $600^{\circ} \mathrm{C}$ to be $47 \mathrm{MPa}$ and $1.9 \times 10^{-9} / \mathrm{sec}$, respectively, based on experimental data [18]. These values are needed to establish $\dot{\varepsilon}_{0}^{*}$ in Equa- tion 3. The coefficient of volume diffusion for $\mathrm{N} 1$ is given by $1.9 \times 10^{-4} \exp (-284 / \mathrm{RT}) \mathrm{m}^{2} \mathrm{sec}^{-1}$, whereas the pipe diffusion coefficient is $3.1 \mathrm{x}$ $10^{-23} \exp (-170 / \mathrm{RT}) \mathrm{m}^{4} \sec ^{-1}$ (4) for the high and low temperature branches respectively. The activation energies are in units of $\mathrm{kJ} / \mathrm{mol}$. For the low temperature branch $\mathrm{m}$ is expected to increase because of the added dependence of the rate of pipe diffusion on $\sigma^{*}$. To include this effect, we used an expression relating the stress, $\sigma$, at SSL creep to the density of dislocations [4]:

$$
\rho=\frac{10}{b^{2}}\left(\frac{\sigma}{G}\right)^{2}
$$

where $b$ is the Burgers' vector. Since $\sigma / \sigma^{*}=0.5$ at SSL creep, Equation 8 may be used to obtain the relationship between $\sigma *$ and $\rho$ :

$$
\rho=\frac{2.5}{b^{2}}\left(\frac{\sigma *}{G}\right)^{2}
$$

The above information is sufficient to construct the dashed curves in Figure 3.

A low rate of workhardening would result in SSL creep. Another mechanism was proposed by Hart [11], whereby SSL creep results from scaling and the nature of Equation 1. Because of the form of Equation 1 and the scaling relationship (Equation 3 ), curves of different hardness are expected to cross at sufficiently low strain rates as depicted in Figure 11. Accordingly, at a stress above the

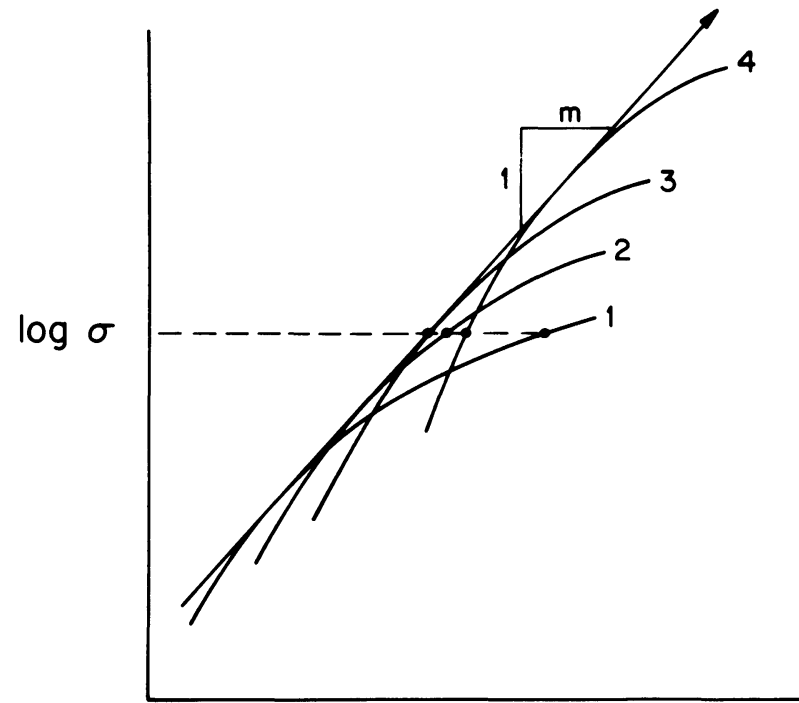

$\log \dot{\epsilon}$

Figure 11. Constant structure flow curves, of the form of Equation 1, displaying the crossing-over behavior created by scaling. The norizontal line represents the mechanical path taken by a specimen during creep under a constant stress. The curves 1-4 are in order of increasing hardness. The creep rate, which is determined by the intersection between the dashed line and the constant hardness curve, is seen to decrease, then increase as the specimen workhardens.

crossing point, a specimen of a higher hardness structure will flow at a lower strain rate (as expected), but at an applied stress below the crossing point, a higher hardness specimen will flow at a higher strain rate contrary to common 
expectation. Because of these characteristics, it is possible that, in a constant load creep test the strain rate will first decrease, then increase, as the material workhardens as indicated in Figure 11. The resulting minimum creep rate might be identified with steady state creep. The minimum strain rate thus obtained would correspond to the condition $\sigma / \sigma^{*}=$ const. $(0.264$ for $m=5)$ which also leads to power law creep. Whether this mechanism operates for the case of nickel is questionable because the stress levels of the high stress portion of the data in Figure 3 are too high for the crossing of the relaxation curves according to measured relaxation data. For the low stress portion of the same data, the rate of workhardening is too low for this criterion to operate.

\section{Additional State Variables}

Up to this point the discussion of flow behavior has pertained only to the homogenous plastic deformation in the grain matrix by dislocation processes at strains in the platic range. Nonelastic deformation often involves other phenomena that require the establishment of additional state variables (if, indeed, such phenomena can be incorporated into a state variable formulation). A number of phenomena that have been categorized include: dislocation anelasticity, microplasticity and the Bauschinger Effect, and grain boundary anelasticity and sliding.

A discussion of these phenomena, how they have been incorporated into a state variable descrip$t$ ion, and how they are examined experimentally have been reviewed in reference [16]. Here, we shall brlefly describe the current view of each.

Whenever a stress is applied to a crystalline solid, the resulting rearrangement of dislocation configurations will lead to a small anelastic strain. Hart [1] proposed that plastic deformation occurs when dislocations at the head of the pileup leak past the barriers confining them, either by thermally induced climb or glide under a sufficiently high applied stress. The driving force for dislocation climb is proportional to the anelastic strain, a, which is a state variable defined by $\partial a=\sigma_{a}$, where $\partial$ is the anelastic

modulus. The value of $\partial$ is determined by the nature of the dislocation configurations.

Microplasticity [19] refers to a collection of phenomena that occur before macroplastic yielding as 11lustrated in Figure 12. In this figure a schematic tensile curve has been expanded to show the stress versus nonelastic strain below about $0.5 \%$ strain. The flow behavior predicted based on a combination of the relationship $\partial a=\sigma_{a}$ and Equation 1 for plastic flow is shown as a solid line, whereas the more realistic material behavior is depicted as a dashed curve. Jackson et a1. [19] Introduced a concept of microplastic flow, that describes better the dashed curve in Figure 12. Physically, the modification requires that two types of dislocation pileups contribute to the stored strain. Long range configurations of dislocations form the first type of pileups against strong barriers. On a more local scale, a second type of pileup is found among weak barriers between the long range barriers as illustrated in Figure 13. Microplasticity results when dislocations leak past the weak barriers to form the long-range pileups responsible for $\sigma_{a}$. The flow

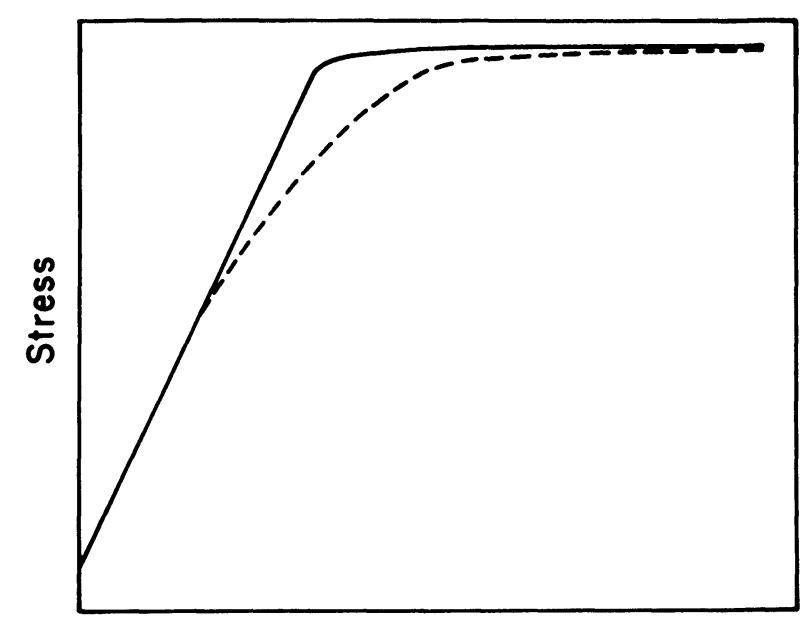

\section{Nonelastic strain}

Figure 12. Initial portion of a tensile curve (< $0.5 \%$ strain) illustrating the behavior containing anelasticity only (solid curve) and real material behavior (dashed curve). The difference between the two behaviors results from the presence of microplasticity.

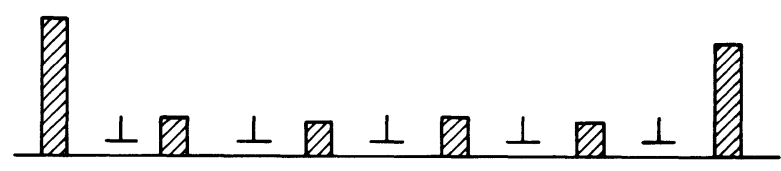

Figure 13. Schematic representation of the barrier structure responsible for microplasticity, as proposed by Jackson [19].

behavior in the microplastic regime can be examined by performing load relaxation tests at the appropriate stress levels. Physically, a distribution of barrier strengths exists. The two barrier model can be considered to be a compromise between physical reality and a sufficiently simple model for which the required parameters can be determined experimentally.

Real materials generally show a Bauschinger effect, in that a specimen which has been plastically deformed, e.g., in compression, exhibits more strain at the same stress in subsequent tensile loading than in compressive reloading. This additional strain can be manifested at quite low stress levels. Hart's model, in which an anelastic element operates in parallel with a glide friction element (Eq. 3) predicts a very weak asymmetry between reloading and reversed loading, that is much less than the observed Bauschinger effect. The microplastic model accounts for the Bauschinger effect better because of the larger amount of stored strains.

At elevated temperatures ( $>0.4 \mathrm{Tm}$ ), grain boundary sliding can become an important mode of deformation $[16,20]$. This process operates simultaneously with grain matrix deformation, but manifests most strongly within a window of strain rate that depends on temperature. In general, the grain boundary sliding rate is less dependent on stress than the grain matrix flow rate. Thus, at strain rates above this window the grain matrix deforms more easily than the grain boundary, and the flow behavior is as described in Equations 1 and 2 . Below this window the grain boundaries 
slide easily but are constrained by grain matrix flow at sites such as triple points, so that the rate of deformation is again controlled by the matrix. At these low strain rates, the grain boundaries act as shear cracks which concentrate the stress at the grain boundary triple points, thereby lowering the stress required to achleve a particular strain rate by a factor of approximately 0.8 [21]. Within the intermediate strain rate window both grain matrix and grain boundary determine the flow behavior. When plotted in a log-log form, flow stress-strain rate data containing the effects of grain boundary sliding will be of a sigmoidal shape. At sufficiently low stresses, grain matrix only deforms elastically and anelastically to accommodate grain boundary sliding, and grain boundary anelasticity results as proposed by Zener [22].

Grain boundary sliding operates within the power law creep regime of the deformation map in Figure 3. To include this additional mode of deformation would only slightly change the character of the curves. However, grain boundary sliding can lead to intergranular fracture that reduces ductility in tensile and creep specimens and therefore is an important subject for study.

\section{Limitations of the State Variable Approach}

Two apparent limitations of state variable approaches w111 be mentioned. They are the description of flow properties of materials containing mobile solutes, sometimes including vacancies, and inhomogenous flow. In many cases inhomogenous flow is known to Involve the interaction of mobile solutes with dislocations [23]; In other cases inhomogenous flow involves processes such as mechanical twinning [24].

During inhomogenous flow, the properties of the specimen can vary spatially as well as with time; therefore, these properties can not be accounted for in terms of a simple state variable description. A range of structural states are expected to exist within the material at one time.

The effects of solute-dislocation interactions, as manifested by phenomena 1ike strain aging, produce. a time-dependence in such parameters as $\dot{\varepsilon} *$ and $\dot{a}$ * during a load relaxation test where $\sigma *$ is nearly constant $[8,25]$. Often this type of the time-dependence is deformation history dependent as we11, so that the strength of the solute-dislocation interaction cannot be specified by current state variables including $\sigma, \dot{\varepsilon}$, and $\sigma *$. Figure 4 depicts such history dependencies. The load re- laxation data shown are produced by a sequence of reloading relaxation experiments [8]. Since the reloading is below the initial stress of the first run, the hardness parameter $\sigma *$ is not expected to change significantly in this series of experiments. It is seen that the reloading data deviate from the inftial data in the direction of lower straln rates suggesting the effect of strain agling. Since the deviation increases as each reloading run is made, the history of reloading is shown to influence strongly the manifestation of solute-dislocation interactions. Whether this type of history dependency can be described by state varlables is not clear at the present.

Summary

A state varlable approach for describing nonelastic deformation in crystalline solids has been reviewed. An 1mportant basis for this approach lies in the determination of flow properties at a constant structure, as done in a load relaxation experiment. When a single mode of deformation (elther dislocation climb or glide) is controlling, the flow stress-strain rate data can be found to translate along a stralght line to overlap one another. This scaling behavlor estab11shes that the 1sostructural data belong to a one-parameter family of curves. This parameter, $\sigma \star$, uniquely determines the flow behavior at a given temperature and a constant structural state.

In addition to the constant structure flow data, a workhardening law representing the evolution of $\sigma^{*}$ with plastic strain is required to predict deformation under arbitrary loading cond1tions. Fortunately, the evolution of $\sigma^{*}$ can be represented in terms of $\sigma^{*}$ and $\sigma_{a}$, so that the flow equations are easily integrable. The integration of the flow equations allows the description of other deformation properties, for instance creep.

Additional state variables are required for phenomena, such as anelasticlty, microplastictity and the Bauschinger effect, and grain boundary sliding and anelasticity. Inhomogenous flow and solute-dislocation interactions are as of yet untreatable by using the present state varlable approach.

\section{Acknowledgment}

Th1s work 1s supported by the U.S. Department of Energy through the Materials Sclence Division.

\section{References}

1. E.W. Hart, J. Eng. Mat, and Tech., 98, p. 193,1976

2. F.H. Huang, H. Yamada and C.-Y. L1, "Const1tutive Relations Based on State Variables for Nonelast1c Deformation in Type 304 and 316 Stainless Steels," in Characterlzation of Materials for Service at Filevated Temperatures, ed. G.V. Smlth, Series MPC-7, ASME, P. 83, 1978.

3. D.W. Henderson, R.C. Kuo, and C.-Y. L1, Scripta Metall., 18, p. 1021, 1984 and F.E. Hauser, Exp. Mech., 6, p. 395, 1966.

4. H.J. Frost and M.F. Äshby, DeformationMechan 1sm Maps, Pergamon Press, Oxford, 1982.
5. M.A. Korhonen, S.-P. Hannula, and C.-Y. L1, Metall. Trans. A, 16A, p. 411, 1985.

6. E.W. Hart and H.D. Solomon, Acta Metall., 21, P. 295, 1973.

7. D. Lee and E.W. Hart, Met. Trans. 2, p. 1245, 1971 .

8. R.-C. Kuo, "Effect of Solute-D1slocation Interactions on the Deformation Behavior of Al$\mathrm{Mg}$ and Fe-C Solld Solut1ons," Ph.D. Thes1s, Cornell Untversity, 1986.

9. P.S. Alexopolous, "An Experimental Invest1gation of Transient Deformation Based on a State Variable Approach," Ph.D. Thesis, Cornell Untversity, 1981. 
10. H. Yamada and C. - Y. LI, Metall. Trans, 4. P. 2133, 1973.

11. E.W. Hart, C.-Y. L1, H. Yamada and G.L. Wire, "Phenomenological Theory: A Gulde to Const1tutive Relations and Fundamental Deformation Propert1es," in Constitutive Equations in Plasticlty, ed. A.S. Argon, MIT Press, Cambridge, Mass., p. 149, 1975.

12. M.A. Korhonen, S.-P. Hannula, and C.-Y. L1, "State Varlable Theorles Based on Hart's Formulation," In Untfled Constitutive Equations for Creep and Plasticlty, ed. A. Miller, Elsevier, Amsterdam, 1987.

13. S.-P. Hannula, M.A. Korhonen, and C.-Y. L1, Res. Mech., 15, P. 99, 1985.

14. G.L. Wire, F.V. E1I1s and C.-Y. L1, Acta Metallurgica, 24, p. 677, 1976.

15. V. Kumar, S. Mukherjee, F.H. Huang and C. $-Y$. L1, "Deformation In Type 304 Austenitic Stainless Steel," Electric Power Research Inst1tute, Report No. EPRI NP-1276, 1979.

16. Che-Yu L1, "State Varlable Theorles for Nonelast1c Deformation," In Metallurgical Treatises, J.R. Tien and J.F. Ell1ott, eds., TMS-AIME, p. $469,1981$.
17. W.D. Jenkins and C.R. Johnson, J. Res. Nat. Bar. Stand., 60, p. 173, 1958.

18. R.L. Keusseyan, "Grain Boundary S11d1ng and Related Phenomena," Ph.D. Thes1s, Cornell Univers1ty, 1985.

19. M.S. Jackson, C. -W. Cho, P. Alexopoulos, and C.-Y. L1, J. Eng. Mat. and Tech., 103, p. 314 , 1981.

20. M.A. Korhonen, R.L. Keusseyan, L. $-X$. L1, and C.-Y. L1, "Load Relaxation Studies of Deformation Mechanisms at Elevated Temperatures. in Novel Techniques in Metal Deformation Testing, R.H. Wagoner, ed., TMS-AIME, P. 275, 1983.

21. F. Ghahreman1, Int. J. Sol1d Structures, $\underline{98}$, p. 193, 1976.

22. C. Zener, Phys. Rev., 60, p. 906, 1941.

23. A.H.Cottre11, Phi1. Mag., 44, P. 829, 1953.

24. F.H. Huang, G.P. Sabol, S.G. McDunald, and C. - Y. L1, J. Nuc. Mat., 79, p. 214, 1979.

25. S.-P. Hannula, R.C. Kuo, D.W. Henderson, and C. - Y. L1, "Load Relaxation Studies of Solute Dislocation Interactions During Strain Aging," in Solute-Defect Interaction, Theory and Experiment, S. Salmoto, G.R. Purdy, and G.V. Kidson, eds., Pergamon, p. 366, 1986. 